\title{
The application of non-invasive and invasive mechanical ventilation in the first episode of acute respiratory failure
}

\author{
Cheng-Chi Huang ${ }^{1,2} \cdot$ Chih-Hsin Muo ${ }^{3} \cdot$ Tain-Fung Wu ${ }^{1} \cdot$ Tung-Yun Chi ${ }^{4} \cdot$ Te-Chun Shen $^{2,5,6} \odot$ - Te-Chun Hsia ${ }^{2}$. \\ Chuen-Ming Shih ${ }^{2}$
}

Received: 29 July 2019 / Accepted: 20 March 2020 / Published online: 30 March 2020

(c) Società Italiana di Medicina Interna (SIMI) 2020

\begin{abstract}
Acute respiratory failure $(\mathrm{RF})$ is a life-threatening syndrome. This study investigated the application of two major clinical strategies, non-invasive mechanical ventilation (NIV) and invasive mechanical ventilation (IMV), in the first episode of acute RF. Data from the longitudinal health insurance database, which included 1,000,000 insured citizens, were used. The NIV group consisted of 1201 patients and the IMV group consisted of 16,072 patients. Chi-square test and $t$ test were applied to determine the differences in categorical and continuous variables. Further analysis was performed by using univariate and multivariable logistic regression and Poisson regression. There was a significant increase of $733 \%$ in the number of NIV users from 2000 to 2012. NIV use was frequently observed in old-age persons (aOR 3.99, 95\% CI 3.06-5.21 for those aged $\geq 80$ years), women (aOR $1.33,95 \%$ CI 1.18-1.50), patients admitted to a high-level hospital (aOR $1.95,95 \%$ CI 1.63-2.34 for those admitted to a medical center), and patients with a higher Charlson comorbidity index (CCI, aOR $1.38-1.66$ for those $\mathrm{CCI} \geq 2$ ). In addition, patients with chronic pulmonary disease, cancer, and congestive heart failure were predominant in NIV users and were significantly associated with NIV use. Overall, the use of NIV has markedly increased over the past few years. Persons of advanced age, women, patients admitted to a high-level hospital, and patients with multiple comorbidities were associated with more frequent NIV use. Chronic pulmonary disease, cancer, and congestive heart failure were most important comorbidities for NIV use.
\end{abstract}

Keywords Acute respiratory failure (acute RF) · Non-invasive mechanical ventilation (NIV) · Invasive mechanical ventilation $(\mathrm{IMV}) \cdot$ Intensive care

Te-Chun Shen and Te-Chun Hsia contributed equally.

Electronic supplementary material The online version of this article (https://doi.org/10.1007/s11739-020-02315-1) contains supplementary material, which is available to authorized users.

Te-Chun Shen

chestshen@gmail.com

1 Department of Business Administration, Asia University, Taichung, Taiwan

2 Division of Pulmonary and Critical Care Medicine, Department of Internal Medicine, China Medical University Hospital, No. 2 Yu-De Road, Taichung 404, Taiwan

3 Management Office for Health Data, China Medical University Hospital, Taichung, Taiwan

\section{Introduction}

Respiratory failure (RF) is a syndrome in which the respiratory system fails to perform one or both of its gas exchange functions, oxygenation and carbon dioxide elimination [1]. $\mathrm{RF}$ can be classified into either acute or chronic according to its clinical course. Acute RF is characterized by life-threatening derangements in arterial blood gases and acid-base

4 Department of Computer Science and Information Management, Hungkuang University, Taichung, Taiwan

5 School of Medicine, China Medical University, Taichung, Taiwan

6 Intensive Care Unit, Chu Shang Show Chwan Hospital, Nantou, Taiwan 
status, and the common etiologies include cardiogenic or noncardiogenic pulmonary edema, pneumonia, and pulmonary hemorrhage (hypoxemic) and drug overdose, neuromuscular disorders, chest wall abnormalities, and obstructive airway disorders (hypercapnic) [2].

The treatment for acute RF is complex and must be immediate. Severe hypoxemic and hypercapnic status must be corrected in time, and the underlying pathophysiologic process that led to acute RF must be identified and managed [3]. Mechanical ventilation (MV) is often performed in this critical situation to assist or replace spontaneous breathing to maintain and sustain life [4]. The invention and application of MV has been considered as one of the cornerstones of modern medicine. MV comprises the following two major clinical settings: non-invasive mechanical ventilation (NIV) and invasive mechanical ventilation (IMV) $[5,6]$.

NIV refers to the administration of ventilatory support without the use of an invasive artificial airway such as an endotracheal tube or a tracheostomy tube. The general indications of NIV for acute RF include acute exacerbation of chronic obstructive pulmonary disease, acute cardiogenic pulmonary edema, asthma, community-acquired pneumonia, post-operative support, and post-extubation support [7, 8]. Other indications of NIV may include palliative care of life, immunocompromised patients, acute respiratory distress syndrome, chest trauma, chest wall and neuromuscular disorders, obesity hypoventilation syndrome, obstructive sleep apnea, and during bronchoscopy [9].

The role of NIV and IMV in acute RF is under vigorous and enthusiastic investigation. The general concept is that NIV is preserved for patients having any of the above-mentioned indications and having no contraindication including the need for airway protection, inability to fit an interface, high severity of illness, or an uncooperative patient [7]. However, cumulative evidence has shown that the use of NIV has markedly increased over the past few decades and that NIV has become an integral tool in the management of acute RF [10-15]. In the present study, we investigated the trend in the use of NIV and IMV, the baseline characteristics of NIV and IMV users, the associated discharge diagnoses of NIV and IMV users, and the mortality of NIV and IMV users during their first episode of acute RF in a population in Taiwan.

\section{Materials and methods}

\section{Data source}

The Taiwan National Health Insurance Administration (TNHIA) had established a single-payer National Health Insurance (NHI) program in 1995. Almost the entire Taiwanese population has to compulsorily participate in this program. The TNHIA established several National Health Insurance Research Databases (NHIRDs) from the NHI program. These databases included information of the insurant and all the medical records of each insurant. The coding of the International Classification of Diseases, Ninth Revision, Clinical Modification (ICD-9-CM) was used for identification of diseases. The identification of insurant in the databases was re-coded according to the Personal Information Protection Act. This study was approved by the Research Ethics Committees of China Medical University and Hospital (CUMH104-REC2-115).

\section{Study population}

Data from the longitudinal health insurance database, which included 1,000,000 insured citizens, were used for this study. We identified all adult patients with acute RF (ICD-9-CM 518.81) from 2000 to 2012. The date of the first acute RF diagnosis was defined as the index date. We excluded inadequate patients as follows: (1) with any diagnosis of RF or use of MV before the index date; (2) without the use of MV for the first episode of acute RF; (3) with overlapping use of NIV and IMV for the first episode of acute RF. All the enrolled patients were further categorized into the following two groups: the NIV group and the IMV group.

\section{Variables of interest}

The variables of interest in the study included age, gender, level of hospital, comorbidity, and Charlson comorbidity index (CCI). Individuals were categorized into the following four age groups: $20-49,50-64,65-79$, and $\geq 80$ years. Hospitals were categorized into the following three levels: medical center, regional hospital, and district hospital. First-level hospitals are medical centers affiliated to colleges of medicine, with high volume of medical personnel and much greater resources with at least 500 beds. Secondlevel hospitals are metropolitan hospitals with at least 250 beds, indicating regional hospitals. Third-level hospitals are local community hospitals with less health care personnel and resources, indicating district hospitals. The CCI was defined based on Charlson's and Devo's reports [16, 17]. The detailed comorbidities included diabetes mellitus, congestive heart failure, myocardial infraction, peripheral vascular disease, cerebrovascular disease, hemiplegia or paraplegia, dementia, chronic pulmonary disease, liver disease, peptic ulcer disease, renal disease, rheumatic disease, cancer, and human immunodeficiency virus infection and acquired immune deficiency syndrome (HIV/AIDS). All comorbidities were identified from 1995 to the index date. In addition, we have analyzed the associated discharge diagnoses 
accompanied by acute RF during the same hospitalization among NIV users. The outcomes of interest included mortality at discharge and mortality within 90 days.

\section{Statistical analysis}

The number of individuals and the probability of the use of NIV and IMV between 2000 and 2012 were calculated. Chi-square test was used to determine the differences in age group, gender, hospital level, comorbidity, and CCI between the NIV group and the IMV group. A $t$ test was carried out to determine the differences in mean age between the two study groups. The odds ratios (ORs) and the $95 \%$ confidence intervals (CIs) for NIV and NIV-associated factors were estimated by logistic regression. The model 1 was adjusted for age group, gender, hospital level, and CCI and the model 2 was adjusted for age group, gender, hospital level, and comorbidities. Furthermore, we calculated the distribution of the most prevalent associated discharge diagnoses in the NIV group and compared with the IMV group by logistic regression. Finally, the rate ratios (RRs) and 95\% CIs of mortality at discharge and within 90 days for the NIV group were compared with those for the IMV group by Poisson regression. The model was adjusted for age group, gender, hospital level, and CCI. Additionally, we performed another overall matched method for mortality comparison (propensity score matching based on age, gender, hospital level, and CCI). All analyses were performed using SAS software (SAS 9.4, SAS Institute, Cary, North Carolina, USA). The level of significance was set at $p<0.05$ of two-tailed tests.

\section{Results}

From the database of $1,000,000$ population, 37,479 individuals were identified to have been diagnosed with acute RF during 2000-2012, of whom 17,273 individuals were enrolled in this study (Supplement data). The NIV group consisting of 1201 (7.0\%) individuals had received NIV treatment, and the IMV group consisting of 16,072 (93.0\%) individuals had received IMV treatment for the first episode of acute RF. We observed that the overall number of MV users increased by $40.6 \%$ from $2000(N=989)$ to 2012 $(N=1391)$ (Fig. 1$)$. The number of NIV users significantly increased by $733 \%$ during this period $(N=27$ in 2000 and $N=225$ in 2012); meanwhile, the number of IMV users increased by only $21.2 \%$ ( $N=962$ in 2000 and $N=1166$ in 2012). The probability of using NIV significantly increased from $2.7 \%$ (27/989) in the year 2000 to $16.2 \%$ (225/1391) in 2012 (Fig. 2).

Compared with IMV users, the NIV users included a greater number of older persons $(84.6 \%$ vs. $69.0 \%$ for those aged $\geq 65$ years), a greater number of women $(45.5 \%$ vs.

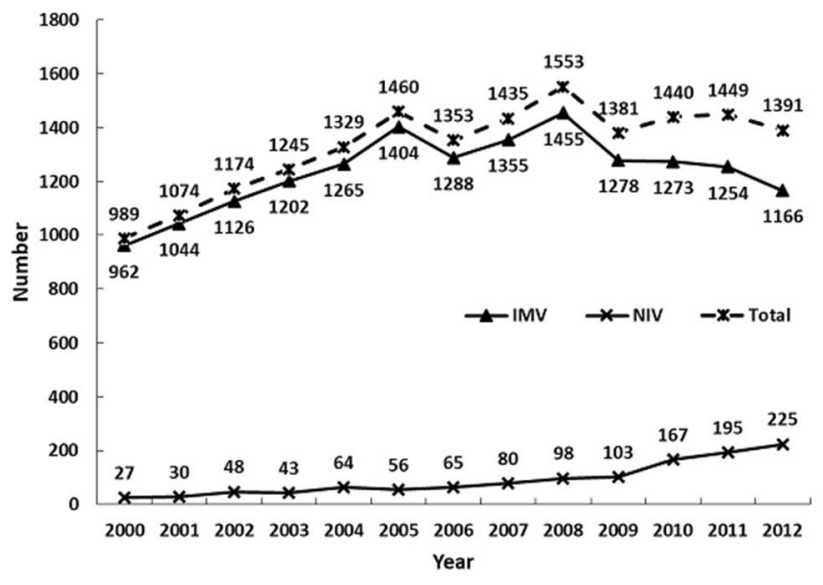

Fig. 1 Individuals in the NIV group and the IMV group between 2000 and 2012

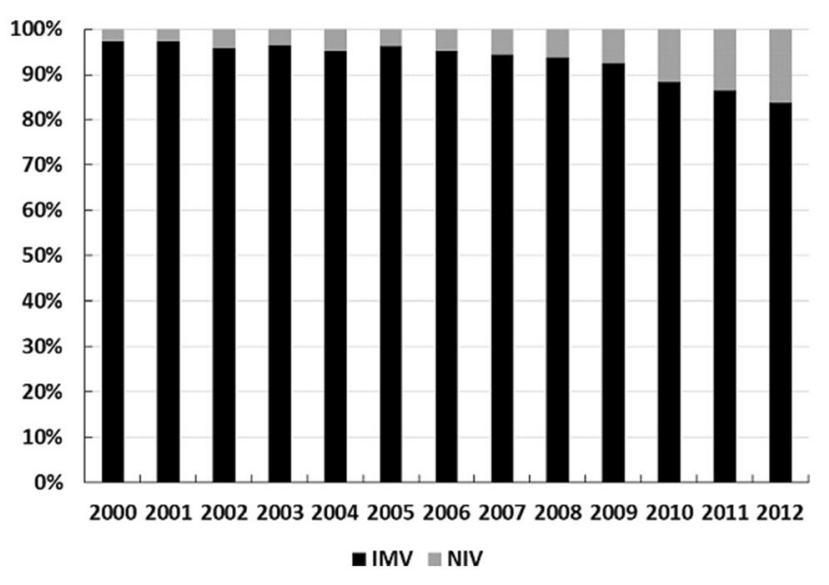

Fig. 2 Probability of the NIV group and the IMV group between 2000 and 2012

$36.9 \%$ ), a greater number of patients admitted to a medical center or a regional hospital ( $84.9 \%$ vs. $78.2 \%$ ), a greater number of patients with a higher CCI $(65.2 \%$ vs. $53.9 \%$ for those $\mathrm{CCI} \geq 2$ ), and a greater number of patients with chronic pulmonary disease $(43.1 \%$ vs. $24.8 \%)$, cancer $(25.8 \%$ vs. $14.0 \%)$, congestive heart failure ( $20.7 \%$ vs. $14.2 \%)$, and dementia (7.74\% vs. $6.17 \%)$. On the other hand, the IMV users included a greater number of patients with renal disease $(12.7 \%$ vs. $8.91 \%)$, diabetes mellitus (9.87\% vs. 7.49 ), liver disease ( $8.89 \%$ vs. $5.25 \%$ ), and peripheral vascular disease $(3.30 \%$ vs. $2.25 \%$ ) than the NIV users (Table 1 ).

Table 2 shows the association between NIV and NIV-associated factors. Compared with patients aged 20-49 years, NIV choice was found with increasing age, but a significant difference was observed only for patients aged $\geq 65$ years (aOR $1.90,95 \%$ CI $1.45-2.49$ at $65-79$ years and aOR $3.99,95 \%$ CI $3.06-5.21$ at $\geq 80$ years 
Table 1 Demographic characteristics and comorbidities in the NIV group and the IMV group

\begin{tabular}{|c|c|c|c|c|c|}
\hline \multirow[t]{3}{*}{ Variables } & \multirow{2}{*}{\multicolumn{2}{|c|}{$\frac{\mathrm{NIV}}{N=1201}$}} & \multirow{2}{*}{\multicolumn{2}{|c|}{$\frac{\mathrm{IMV}}{N=16,072}$}} & \multirow[t]{3}{*}{$p$ value } \\
\hline & & & & & \\
\hline & $N$ & $\%$ & $N$ & $\%$ & \\
\hline \multicolumn{5}{|l|}{ Age group } & $<0.0001$ \\
\hline $20-49$ & 64 & 5.33 & 2074 & 12.9 & \\
\hline $50-64$ & 120 & 9.99 & 2914 & 18.1 & \\
\hline $65-79$ & 392 & 32.6 & 6231 & 38.8 & \\
\hline$\geq 80$ & 625 & 52.0 & 4853 & 30.2 & \\
\hline Mean $(\mathrm{SD})^{\mathrm{a}}$ & 77.5 & $(13.5)$ & 70.1 & $(15.6)$ & $<0.0001$ \\
\hline \multicolumn{5}{|l|}{ Gender } & $<0.0001$ \\
\hline Women & 546 & 45.5 & 5924 & 36.9 & \\
\hline Men & 655 & 54.5 & 10,148 & 63.1 & \\
\hline \multicolumn{5}{|l|}{ Hospital level } & $<0.0001$ \\
\hline District hospital & 182 & 15.2 & 3508 & 21.8 & \\
\hline Regional hospital & 576 & 48.0 & 6870 & 42.8 & \\
\hline Medical center & 443 & 36.9 & 5694 & 35.4 & \\
\hline \multicolumn{6}{|l|}{ Comorbidity } \\
\hline Diabetes mellitus & 90 & 7.49 & 1576 & 9.81 & 0.009 \\
\hline Congestive heart failure & 248 & 20.7 & 2284 & 14.2 & $<0.0001$ \\
\hline Myocardial infarction & 50 & 4.16 & 760 & 4.73 & 0.37 \\
\hline $\begin{array}{l}\text { Peripheral vascular } \\
\text { disease }\end{array}$ & 27 & 2.25 & 531 & 3.30 & 0.046 \\
\hline Cerebrovascular disease & 341 & 28.4 & 4547 & 28.3 & 0.94 \\
\hline Hemiplegia or paraplegia & 80 & 6.66 & 1040 & 6.47 & 0.80 \\
\hline Dementia & 93 & 7.74 & 992 & 6.17 & 0.03 \\
\hline $\begin{array}{l}\text { Chronic pulmonary } \\
\text { disease }\end{array}$ & 518 & 43.1 & 3990 & 24.8 & $<0.0001$ \\
\hline Liver disease & 63 & 5.25 & 1428 & 8.89 & $<0.0001$ \\
\hline Peptic ulcer disease & 303 & 25.2 & 3929 & 24.5 & 0.54 \\
\hline Renal disease & 107 & 8.91 & 2047 & 12.7 & 0.0001 \\
\hline Rheumatic disease & 12 & 1.00 & 152 & 0.95 & 0.85 \\
\hline Cancer & 310 & 25.8 & 2252 & 14.0 & $<0.0001$ \\
\hline AIDS/HIV & 1 & 0.08 & 14 & 0.09 & 0.97 \\
\hline \multicolumn{5}{|l|}{ Charlson comorbidity index } & $<0.0001$ \\
\hline $0-1$ & 418 & 34.8 & 7401 & 46.1 & \\
\hline $2-3$ & 493 & 41.1 & 5070 & 31.6 & \\
\hline $4-5$ & 191 & 15.9 & 2344 & 14.6 & \\
\hline$>5$ & 99 & 8.24 & 1257 & 7.82 & \\
\hline
\end{tabular}

AIDS acquired immune deficiency syndrome, HIV human immunodeficiency virus, $I M V$ invasive mechanical ventilation, $N I V$ non-invasive mechanical ventilation, $S D$ standard deviation;

Chi-square test and ${ }^{\mathrm{a}} t$ test

in the model 1). Women had a 1.33-fold NIV choice compared to that of men (95\% CI 1.18-1.50 in the model 1). Compared with patients admitted to a district hospital, those who were admitted to a regional hospital or a medical center had an about 1.9-fold NIV choice in the model 1. Compared with patients with CCI at $0-1$, the highest NIV choice was at
2-3 (aOR 1.66, 95\% CI 1.45-1.90) and followed by at $>5$ (aOR 1.40, 95\% CI 1.11-1.77) and at 4-5 (aOR 1.38, 95\% CI 1.15-1.65). In the model 2, there were the same trend in age group, gender, and hospital level as in the model 1. Patients comorbid with a medical history of cancer (aOR $2.57,95 \%$ CI 2.23-2.97), chronic pulmonary disease (aOR $2.24,95 \%$ CI $1.97-2.55$ ), and congestive heart failure (aOR $1.27,95 \%$ CI 1.08-1.49) had a significantly higher NIV choice compared to that of individuals without those medical history. In contrast, patients comorbid with a medical history of renal disease (aOR 0.59, 95\% CI 0.48-0.74) and liver disease (aOR $0.65,95 \%$ CI $0.50-0.85$ ) had a significantly lower NIV choice. In addition, we also presented the association between NIV and NIV-associated factors stratified by (presence or absence of) cancer history. The most notable finding was that the impact of increasing age on NIV choice was weakened (aOR 1, 0.92, 1.20, and 2.06 in model 1 and aOR $1,0.91,1.06,1.62$ in model 2) in patients with a history of cancer but strengthened (aOR 1, 1.37, 2.80, and 6.53 in model 1 and aOR 1, 1.32, 2.36, 5.11 in model 2) in patients with no cancer history (Supplementary data).

Table 3 shows the most frequent associated discharge diagnoses with acute RF in the NIV group (No. $=22,>2 \%$ ). We categorized these associated disorders into four groups: (1) possible cause of RF, (2) comorbidity, (3) co-existing acute illness, and (4) uncertain acute or chronic condition. Pneumonia (aOR 1.44, 95\% CI 1.27-1.62); cancer (aOR 2.40, 95\% CI 2.08-2.77); chronic obstructive pulmonary disease (aOR 2.73, 95\% CI 2.34-3.19); heart failure (aOR 1.25, 95\% CI 1.05-1.50); hypertensive heart disease (aOR 1.36, 95\% CI 1.13-1.64); fluid, electrolyte, and acid-base imbalance (aOR 1.66, 95\% CI 1.35-2.04); pleurisy (aOR 1.66, 95\% CI 1.30-2.11); asthma (aOR 2.72, 95\% CI 2.08-3.58); and bronchiectasis (aOR 5.75, 95\% CI 3.76-8.80) were significantly associated with a high NIV choice. By contrast, septicemia (aOR 0.76, 95\% CI 0.66-0.87), shock (aOR $0.56,95 \%$ CI 0.47-0.66), acute renal failure (aOR 0.73, 95\% CI 0.58-0.91), cardiac dysrhythmias (aOR 0.75, 95\% CI 0.58-0.97), pneumonitis due to solids and liquids (aOR $0.62,95 \%$ CI $0.47-0.82$ ), gastrointestinal hemorrhage (aOR $0.71,95 \%$ CI 0.52-0.96), chronic kidney disease (aOR 0.64, 95\% CI 0.46-0.87), and acute myocardial infarction (aOR $0.40,95 \%$ CI $0.27-0.60$ ) were significantly associated with a low NIV choice.

Furthermore, we observed that patients in the NIV group had higher overall mortality rate at discharge $(57.8 \%$ vs. $47.9 \%)$ and within 90 days $(69.0 \%$ vs. $59.5 \%)$ than the patients in the IMV group, with the adjusted RRs being 1.15 (95\% CI 1.07-1.25) and 1.11 (95\% CI 1.04-1.20), respectively (upper part in Table 4). Stratified analysis for the presence of cancer history showed that patients with cancer in the NIV group had a higher mortality rate at discharge (77.4\% vs. $62.6 \%)$ and within 90 days $(89.7 \%$ vs. 
Table 2 Odds ratios and 95\% confidence intervals for NIV and NIV-associated factors

\begin{tabular}{|c|c|c|c|}
\hline Variables & $\begin{array}{l}\text { Crude } \\
\text { OR }(95 \% \mathrm{CI})\end{array}$ & $\begin{array}{l}\text { Adjusted }^{\mathrm{a}} \\
\text { OR }(95 \% \mathrm{CI})\end{array}$ & $\begin{array}{l}\text { Adjusted }^{\mathrm{b}} \\
\text { OR }(95 \% \mathrm{CI})\end{array}$ \\
\hline \multicolumn{4}{|l|}{ Age group } \\
\hline $20-49$ & 1 & 1 & 1 \\
\hline $50-64$ & $1.33(0.98-1.82)$ & $1.22(0.90-1.67)$ & $1.17(0.85-1.59)$ \\
\hline $65-79$ & $2.04(1.56-2.67)^{* * *}$ & $1.90(1.45-2.49)^{* * *}$ & $1.70(1.29-2.24)^{* * *}$ \\
\hline$\geq 80$ & $4.17(3.21-5.42)^{* * *}$ & $3.99(3.06-5.21)^{* * *}$ & $3.49(2.66-4.58)^{* * *}$ \\
\hline \multicolumn{4}{|l|}{ Gender } \\
\hline Women & $1.43(1.27-1.61)^{* * *}$ & $1.33(1.18-1.50)^{* * *}$ & $1.48(1.31-1.67)^{* * *}$ \\
\hline Men & 1 & 1 & 1 \\
\hline \multicolumn{4}{|l|}{ Hospital level } \\
\hline District hospital & 1 & 1 & 1 \\
\hline Regional hospital & $1.62(1.36-1.92)^{* * *}$ & $1.86(1.57-2.22)^{* * *}$ & $1.92(1.61-2.29)^{* * * *}$ \\
\hline Medical center & $1.50(1.26-1.79)^{* * *}$ & $1.95(1.63-2.34)^{* * *}$ & $1.97(1.64-2.38)^{* * *}$ \\
\hline \multicolumn{4}{|l|}{ Charlson comorbidity index } \\
\hline $0-1$ & 1 & 1 & \\
\hline $2-3$ & $1.72(1.50-1.97)^{* * *}$ & $1.66(1.45-1.90)^{* * *}$ & \\
\hline $4-5$ & $1.44(1.21-1.72)^{* * *}$ & $1.38(1.15-1.65)^{* * *}$ & \\
\hline$>5$ & $1.39(1.11-1.75)^{* *}$ & $1.40(1.11-1.77)^{* * *}$ & \\
\hline \multicolumn{4}{|l|}{ Comorbidity (yes vs. no) } \\
\hline Diabetes mellitus & $0.75(0.60-0.93)^{* *}$ & & $0.91(0.72-1.15)$ \\
\hline Congestive heart failure & $1.57(1.36-1.82)^{* * *}$ & & $1.27(1.08-1.49)^{* *}$ \\
\hline Myocardial infarction & $0.88(0.65-1.17)$ & & \\
\hline Peripheral vascular disease & $0.67(0.46-0.99)^{*}$ & & $0.71(0.47-1.07)$ \\
\hline Cerebrovascular disease & $1.01(0.88-1.15)$ & & \\
\hline Hemiplegia or paraplegia & $1.03(0.82-1.31)$ & & \\
\hline Dementia & $1.28(1.02-1.59)^{*}$ & & $0.97(0.77-1.23)$ \\
\hline Chronic pulmonary disease & $2.30(2.04-2.59) * * *$ & & $2.24(1.97-2.55)^{* * *}$ \\
\hline Liver disease & $0.57(0.4-0.74)^{* * *}$ & & $0.65(0.50-0.85)^{* *}$ \\
\hline Peptic ulcer disease & $1.04(0.91-1.19)$ & & \\
\hline Renal disease & $0.67(0.55-0.82)^{* * *}$ & & $0.59(0.48-0.74)^{* * *}$ \\
\hline Rheumatic disease & $1.06(0.59-1.91)$ & & \\
\hline Cancer & $2.14(1.86-2.45)^{* * *}$ & & $2.57(2.23-2.97)^{* * *}$ \\
\hline AIDS/HIV & $0.96(0.13-7.28)$ & & \\
\hline
\end{tabular}

AIDS acquired immune deficiency syndrome, $C I$ confidence intervals, $H I V$ human immunodeficiency virus, NIV non-invasive mechanical ventilation, $O R$ odds ratio

$* p<0.05$

$* * p<0.01$

$* * * p<0.001$

${ }^{a}$ Model 1 was adjusted for age group, gender, hospital level, and Charlson comorbidity index

${ }^{b}$ Model 2 was adjusted for age group, gender, hospital level, and comorbidity which with a significant difference in crude model
$76.2 \%)$ than patients with cancer in the IMV group. However, no difference was observed in the mortality rate of non-cancer patients who had received NIV and IMV treatment. To validate the results, we additionally performed propensity score matching based on age, gender, hospital level, and CCI for mortality comparison $(N=1188$ in NIV group and $N=4752$ in IMV group, lower part in Table 4).
Similarly, we observed that patients in the NIV group had a significantly higher overall mortality rate at discharge than those in the IMV group. Patients with cancer in the NIV group had a higher mortality rate at discharge and within 90 days than patients with cancer in the IMV group. No difference was observed in the mortality rate of noncancer patients who had received NIV and IMV treatment. 
Table 3 Top associated discharge diagnoses ( $>2 \%$ ) during the same hospitalization in the NIV group and compared with the IMV group

\begin{tabular}{|c|c|c|c|c|c|c|}
\hline \multirow[t]{2}{*}{ Associated discharge diagnoses (ICD-9 code) } & \multicolumn{2}{|l|}{ NIV } & \multicolumn{2}{|l|}{ IMV } & \multirow{2}{*}{$\begin{array}{l}\text { Crude } \\
\text { OR }(95 \% \mathrm{CI})\end{array}$} & \multirow{2}{*}{$\begin{array}{l}\text { Adjusted }^{\mathrm{a}} \\
\text { OR }(95 \% \mathrm{CI})\end{array}$} \\
\hline & $N$ & $\%$ & $N$ & $\%$ & & \\
\hline \multicolumn{7}{|l|}{ Possible cause of RF } \\
\hline Pneumonia $(482,486)$ & 603 & 50.2 & 6191 & 38.5 & $1.61(1.43-1.81)^{* * *}$ & $1.44(1.27-1.62)^{* * *}$ \\
\hline Chronic obstructive pulmonary disease $(491,496)$ & 264 & 22.0 & 1459 & 9.08 & $2.82(2.44-3.27) * * *$ & $2.73(2.34-3.19)^{* * *}$ \\
\hline Asthma (493) & 68 & 5.66 & 406 & 2.53 & $2.32(1.78-3.02)^{* * *}$ & $2.72(2.08-3.58)^{* * *}$ \\
\hline Pneumonitis due to solids and liquids (507) & 58 & 4.83 & 1097 & 6.83 & $0.69(0.53-0.91)^{* *}$ & $0.62(0.47-0.82)^{* * *}$ \\
\hline Bronchiectasis (494) & 33 & 2.75 & 77 & 0.48 & $5.87(3.89-8.87)^{* * *}$ & $5.75(3.76-8.80)^{* * *}$ \\
\hline \multicolumn{7}{|l|}{ Comorbidity } \\
\hline Malignancy (140-208) & 310 & 25.8 & 2281 & 14.2 & $2.10(1.84-2.41) * * *$ & $2.40(2.08-2.77)^{* * *}$ \\
\hline Diabetes mellitus (250) & 188 & 15.7 & 2701 & 16.8 & $0.92(0.78-1.08)$ & $0.88(0.75-1.04)$ \\
\hline Hypertensive heart disease $(401,402)$ & 142 & 11.8 & 1413 & 8.79 & $1.39(1.16-1.67)^{* * *}$ & $1.36(1.13-1.64)^{* *}$ \\
\hline Other forms of chronic ischemic heart disease (414) & 52 & 4.33 & 686 & 4.27 & $1.02(0.76-1.35)$ & $0.90(0.67-1.20)$ \\
\hline Chronic kidney disease (585) & 44 & 3.66 & 822 & 5.11 & $0.71(0.52-0.96)^{*}$ & $0.64(0.46-0.87)^{* *}$ \\
\hline Chronic liver disease and cirrhosis (571) & 39 & 3.25 & 859 & 5.34 & $0.59(0.43-0.82)^{* *}$ & $0.82(0.59-1.15)$ \\
\hline \multicolumn{7}{|l|}{ Co-existing acute illness } \\
\hline Septicemia (038) & 310 & 25.8 & 4753 & 29.6 & $0.83(0.73-0.95)^{* *}$ & $0.76(0.66-0.87)^{* * *}$ \\
\hline Urinary tract infection (599.0) & 173 & 14.4 & 1838 & 11.4 & $1.30(1.01-1.54)^{* *}$ & $1.06(0.90-1.27)$ \\
\hline Shock (785.5) & 158 & 13.2 & 3266 & 20.3 & $0.59(0.50-0.71) * * *$ & $0.56(0.47-0.66)^{* * *}$ \\
\hline Fluid, electrolyte, and acid-base imbalance (276) & 115 & 9.58 & 972 & 6.05 & $1.65(1.34-2.02)^{* * *}$ & $1.66(1.35-2.04)^{* * *}$ \\
\hline Acute renal failure (584) & 90 & 7.49 & 1519 & 9.45 & $0.78(0.62-0.97)^{*}$ & $0.73(0.58-0.91)^{* *}$ \\
\hline Gastrointestinal hemorrhage (578) & 47 & 3.91 & 856 & 5.33 & $0.72(0.54-0.98)^{*}$ & $0.71(0.52-0.96)^{*}$ \\
\hline Acute myocardial infarction (410) & 25 & 2.08 & 715 & 4.45 & $0.46(0.31-0.68)^{* * *}$ & $0.40(0.27-0.60)^{* * *}$ \\
\hline \multicolumn{7}{|l|}{ Uncertain acute or chronic condition } \\
\hline Heart failure (428) & 161 & 13.4 & 1489 & 9.26 & $1.52(1.27-1.81)^{* * *}$ & $1.25(1.05-1.50)^{*}$ \\
\hline Pleurisy (511) & 82 & 6.83 & 598 & 3.72 & $1.90(1.49-2.41)^{* * *}$ & $1.66(1.30-2.11)^{* * *}$ \\
\hline Cardiac dysrhythmias (427) & 68 & 5.66 & 1106 & 6.88 & $0.81(0.63-1.05)$ & $0.75(0.58-0.97)^{*}$ \\
\hline Occlusion of cerebral arteries (434) & 43 & 3.58 & 689 & 4.29 & $0.83(0.61-1.14)$ & $0.78(0.57-1.07)$ \\
\hline
\end{tabular}

$I C D$-9 international classification of diseases, ninth revision, $I M V$ invasive mechanical ventilation, $N I V$ non-invasive mechanical ventilation, $R F$ respiratory failure

$* p<0.05$

$* * p<0.01$

$* * * p<0.001$

${ }^{a}$ Model was adjusted for age group, gender, hospital level, and Charlson comorbidity index

\section{Discussion}

This work is a large population-based study evaluating the application of NIV and IMV in the first episode of acute RF. We observed that the use of NIV has markedly increased over the past few years, which implies that NIV application has largely expanded. Characteristic analysis of the study population showed that the elderly, women, patients admitted to a high-level hospital, and patients with a high CCI were more predominant users of NIV than their counterparts. We also found that individuals who had received NIV treatment had a higher mortality rate than those who had received IMV treatment; however, there was no difference in the mortality rate of non-cancer individuals who had received NIV treatment in comparison with those who had received IMV treatment.

In the present study, we have provided valuable information about underlying comorbidities among patients with acute RF. We found that cancer, chronic pulmonary disease, and congestive heart failure were associated with a high NIV choice, whereas renal and liver diseases were associated with a low NIV choice. In addition, we have provided the most frequent associated discharge diagnoses among NIV users and the influence of these associated discharge diagnoses on the use of NIV. These results are consistent with the general concepts of NIV and IMV indications.

Cumulative evidence has shown that the use of NIV has markedly increased over the past few decades. In a recent review, NIV is suggested for application in broad conditions 
Table 4 Mortality at discharge and within 90 days in the NIV group and in the IMV group

\begin{tabular}{|c|c|c|c|c|c|c|}
\hline & \multicolumn{2}{|l|}{ NIV } & \multicolumn{2}{|l|}{ IMV } & \multirow[t]{2}{*}{ Crude RR (95\% CI) } & \multirow[t]{2}{*}{ Adjusted RR $(95 \% \mathrm{CI})$} \\
\hline & $N$ & Death no. $(\%)$ & $N$ & Death no. $(\%)$ & & \\
\hline \multicolumn{7}{|c|}{ Without matching } \\
\hline \multicolumn{7}{|l|}{ At discharge } \\
\hline Overall & 1201 & $694(57.8)$ & 16,072 & $7700(47.9)$ & $1.21(1.12-1.30)^{* * *}$ & $1.15(1.07-1.25)^{* * *}$ \\
\hline Cancer & 310 & $240(77.4)$ & 2252 & $1410(62.6)$ & $1.24(1.08-1.42)^{* *}$ & $1.24(1.08-1.42)^{* *}$ \\
\hline Non-cancer & 891 & $454(51.0)$ & 13,820 & $6290(45.5)$ & $1.12(1.02-1.23)^{*}$ & $1.05(0.95-1.15)$ \\
\hline \multicolumn{7}{|l|}{ Within 90 days } \\
\hline Overall & 1201 & $829(69.0)$ & 16,072 & $9562(59.5)$ & $1.16(1.08-1.25)^{* * *}$ & $1.11(1.04-1.20)^{* *}$ \\
\hline Cancer & 310 & $278(89.7)$ & 2252 & $1715(76.2)$ & $1.18(1.04-1.34)^{*}$ & $1.18(1.04-1.34)^{*}$ \\
\hline Non-cancer & 891 & $551(61.8)$ & 13,820 & $7847(56.8)$ & $1.09(0.99-1.19)$ & $1.02(0.94-1.12)$ \\
\hline \multicolumn{7}{|l|}{ With matching ${ }^{\mathrm{a}}$} \\
\hline \multicolumn{7}{|l|}{ At discharge } \\
\hline Overall & 1188 & $686(57.7)$ & 4752 & $2453(51.6)$ & $1.12(1.03-1.22)^{* *}$ & $1.12(1.02-1.21)^{*}$ \\
\hline Cancer & 310 & $240(77.4)$ & 1240 & $772(62.3)$ & $1.24(1.08-1.44)^{* *}$ & $1.24(1.08-1.44)^{* *}$ \\
\hline Non-cancer & 878 & $446(50.8)$ & 3512 & $1681(47.9)$ & $1.06(0.96-1.18)$ & $1.06(0.95-1.17)$ \\
\hline \multicolumn{7}{|l|}{ Within 90 days } \\
\hline Overall & 1188 & $819(68.9)$ & 4752 & $3051(64.2)$ & $1.07(0.99-1.16)$ & $1.07(0.99-1.16)$ \\
\hline Cancer & 310 & $278(89.7)$ & 1240 & $935(75.4)$ & $1.19(1.04-1.36)^{*}$ & $1.19(1.04-1.36)^{*}$ \\
\hline Non-cancer & 878 & $541(61.6)$ & 3512 & $2116(60.3)$ & $1.02(0.93-1.12)$ & $1.02(0.93-1.12)$ \\
\hline
\end{tabular}

$C I$ confidence intervals, $I M V$ invasive mechanical ventilation, $N I V$ non-invasive mechanical ventilation, $R R$ rate ratio

${ }^{*} p<0.05$

$* * p<0.01$

$* * * p<0.001$

a Propensity score matching for age group, gender, hospital level, and Charlson comorbidity index

${ }^{\mathrm{b}}$ Model was adjusted for age group, gender, hospital level, and Charlson comorbidity index

in acute RF: (1) to prevent endotracheal intubation; (2) as an alternative to IMV; (3) as a tool for facilitating early weaning from IMV; or (4) as palliative care in do not resuscitate (DNR) patients [18]. The indications for NIV have also been extended, such as for immunocompromised patients and patients with chest trauma, post-operative lung resection, community-acquired pneumonia, acute respiratory distress syndrome, and terminal stage diseases (palliative care) [7-9, 18]. The improvement of intensive monitoring and care, the application of integrated therapeutic tools, and expert integrated teams all contribute to the increased use of NIV.

Among the abovementioned indications for NIV, palliative use may contribute an important role for NIV application. In this study, the extremely elderly and patients with cancer comprised a very high proportion $(52.0 \%$ of those aged $\geq 80$ years and $25.8 \%$ of those with a history of cancer) of NIV users, and cancer occupied the second place among the associated discharge diagnoses in NIV users. This phenomenon may be influenced by the administration of palliative care for individuals with extremely old age and advanced stage of cancer [19-22]. Palliative NIV could be administered either to offer a chance for survival or to alleviate the symptoms of respiratory distress in terminally ill patients [19]. The use of NIV in patients with dyspnea in terminal states remains controversial, but it is reportedly effective in reducing dyspnea and in decreasing the dose of morphine in palliative use in patients with end-stage cancer [21]. One of the primary benefits of using NIV in this setting is the preservation of communication between the patient and the family. This technique is also widely used in patients with acute RF and in a DNR order, particularly for the extremely elderly [22]. Unfortunately, information on the number of persons who had DNR orders or received hospice care was absent from the database. The role or exact proportion of palliative NIV in all NIV users should be clarified in further research.

In this study, the overall mortality was higher in the NIV group than in the IMV group. In general, NIV is applied for patients with low disease severity; therefore, we believe that palliative NIV plays a vital role in clinical practice. We further conducted a stratified analysis of the presence of cancer history and found that patients with cancer who had received NIV treatment had a much higher mortality rate than patients who had received IMV treatment. By contrast, no difference was found in the mortality rates of patients without cancer between the NIV and IMV 
groups. This finding supported our hypothesis because palliative NIV was applied in a considerable number of patients with and without cancer. However, the exact proportion of palliative NIV users in this study was unknown. The application of palliative NIV for non-cancer patients needs further investigation.

The strength of this study is that it is a nationwide population-based investigation evaluating the application of NIV and IMV in the first episode of acute RF. The NHI program in Taiwan covers more than $99.5 \%$ of the population. This universal coverage has reduced the barriers to healthcare access for all citizens, irrespective of the socioeconomic background and the residential location [23]. In the present study, a real-world scenario has been depicted, wherein all diagnoses and medical practices were directly made during a medical consultation.

Several limitations were considered in this study. First, we evaluated the role of NIV and IMV only in the first episode of acute RF. Some people may have repeated episodes of acute RF and would have received different opinions regarding treatment. Second, we excluded the overlapping use of NIV and IMV as we could not differentiate the time sequence of NIV and IMV use in the database. Clinically, IMV can be used after NIV for the management of disease progression, and NIV can be used after IMV for post-extubation support. Third, the cause of acute RF was unavailable in the database. We attempted to apply associated discharge diagnoses to overcome this limitation, but only minimal success was achieved. We could only categorize these associated diagnoses into four groups: possible cause of RF, comorbidity, co-existing acute illness, and uncertain acute or chronic condition. Fourth, disease severity such as the degree of hypoxemia and hypercapnia and the acute physiology and chronic health evaluation (APACHE) score were unavailable. The APACHE score is associated with patients' prognosis and in-hospital mortality [24]. We could only collect CCI data to represent the overall degree of severity. Finally, we were unable to identify patients with DNR orders or who received palliative and hospice care. Therefore, the results of the present study must be interpreted with caution.

\section{Conclusion}

The use of NIV has markedly increased over the past few years. Persons of advanced age, women, patients admitted to a high-level hospital, and patients with multiple comorbidities were associated with more frequent NIV use. Chronic pulmonary disease, cancer and congestive heart failure were predominant in NIV users and were significantly associated with NIV use.
Funding This study is supported by China Medical University Hospital (DMR-108-110) and Taiwan Ministry of Health and Welfare (MOHW109-TDU-B-212-114004).

\section{Compliance with ethical standards}

Conflict of interest The authors declare that they have no conflict of interest.

Statement of human and animal rights This article does not contain any studies with human participants or animals performed by any of the authors.

Informed consent Not necessary, all patient information in the NHIRD was anonymized and de-identified prior to analysis.

\section{References}

1. Roussos C, Koutsoukou A (2003) Respiratory failure. Eur Respir J Suppl 47:3s-14s

2. Schneider J, Sweberg T (2013) Acute respiratory failure. Crit Care Clin 29:167-183

3. Fry AC, Farrington K (2006) Management of acute renal failure. Postgrad Med J 82:106-116

4. Marini JJ (2013) Mechanical ventilation: past lessons and the near future. Crit Care 17(Suppl 1):S1

5. Pierson DJ (2002) Indications for mechanical ventilation in adults with acute respiratory failure. Respir Care 47:249-262

6. Brochard L (2003) Mechanical ventilation: invasive versus noninvasive. Eur Respir J Suppl 47:31s-37s

7. Hess DR (2013) Noninvasive ventilation for acute respiratory failure. Respir Care 58:950-972

8. Singh G, Pitoyo CW (2014) Non-invasive ventilation in acute respiratory failure. Acta Med Indones 46:74-80

9. Mas A, Masip J (2014) Noninvasive ventilation in acute respiratory failure. Int J Chron Obstruct Pulmon Dis 9:837-852

10. Caples SM, Gay PC (2005) Noninvasive positive pressure ventilation in the intensive care unit: a concise review. Crit Care Med 33:2651-2658

11. Garpestad E, Brennan J, Hill NS (2007) Noninvasive ventilation for critical care. Chest 132:711-720

12. Hill NS, Brennan J, Garpestad E, Nava S (2007) Noninvasive ventilation in acute respiratory failure. Crit Care Med 35:2402-2407

13. Keenan SP, Mehta S (2009) Noninvasive ventilation for patients presenting with acute respiratory failure: the randomized controlled trials. Respir Care 54:116-126

14. Nava S, Hill N (2009) Non-invasive ventilation in acute respiratory failure. Lancet 374:250-259

15. Demoule A, Chevret S, Carlucci A, Kouatchet A, Jaber S, Meziani $\mathrm{F}$ et al (2016) Changing use of noninvasive ventilation in critically ill patients: trends over 15 years in francophone countries. Intensive Care Med 42:82-92

16. Charlson ME, Pompei P, Ales KL, MacKenzie CR (1987) A new method of classifying prognostic comorbidity in longitudinal studies: Development and validation. J Chronic Dis 40:373-383

17. Deyo RA, Cherkin DC, Ciol MA (1992) Adapting a clinical comorbidity index for use with ICD-9-CM administrative databases. J Clin Epidemiol 45:613-619

18. Scala R, Pisani L (2018) Noninvasive ventilation in acute respiratory failure: Which recipe for success? Eur Respir Rev 27:180029 
19. Azoulay E, Demoule A, Jaber S, Kouatchet A, Meert AP, Papazian L et al (2011) Palliative noninvasive ventilation in patients with acute respiratory failure. Intensive Care Med 37:1250-1257

20. Cuomo A, Delmastro M, Ceriana P, Nava S, Conti G, Antonelli $M$ et al (2004) Noninvasive mechanical ventilation as a palliative treatment of acute respiratory failure in patients with end-stage solid cancer. Palliat Med 18:602-610

21. Nava S, Ferrer M, Esquinas A, Scala R, Groff P, Cosentini R et al (2013) Palliative use of non-invasive ventilation in end-of-life patients with solid tumours: a randomised feasibility trial. Lancet Oncol 14:219-227

22. Piroddi IMG, Barlascini C, Esquinas A, Braido F, Banfi P, Nicolini A (2017) Non-invasive mechanical ventilation in elderly patients: a narrative review. Geriatr Gerontol Int 17:689-696
23. Hsing AW, Ioannidis JP (2015) Nationwide population science: lessons from the Taiwan National Health Insurance Research Database. JAMA Intern Med 175:1527-1529

24. Michael SS, Reznek MA (2019) Comparison of risk-adjustment methods to predict in-hospital mortality among emergency department patients admitted to critical care settings. J Emerg Crit Care Med 3:9

Publisher's Note Springer Nature remains neutral with regard to jurisdictional claims in published maps and institutional affiliations. 